\title{
ANÁlISE DE SÉRIES TEMPORAIS NA OPERAÇÃO DE SISTEMA DE TRATAMENTO DE ÁGUAS RESIDUÁRIAS DE ABATEDOURO DE FRANGO
}

\section{TIME SERIES ANALYSIS ON THE OPERATION OF A POULTRY SLAUGHTERHOUSE WASTEWATER TREATMENT PLANT}

\section{IVANA RIBEIRO DE NARDI}

Doutora em Hidráulica e Saneamento pela Escola de Engenharia de São Carlos, Universidade de São Paulo. Pesquisadora no Centro Universitário Central Paulista

\section{ALMIR ROGÉRIO DE LIMA}

Graduando em Administração de Empresas do Centro Universitário Central Paulista

\section{ARIUSKa KARLA BARBOSA AMORIM}

Doutora em Hidráulica e Saneamento pela Escola de Engenharia de São Carlos, Universidade de São Paulo. Pesquisadora no Centro Universitário Central Paulista

\section{VALÉRIA DEL NERY}

Doutora em Hidráulica e Saneamento pela Escola de Engenharia de São Carlos, Universidade de São Paulo. Consultora na área de tratamento de águas residuárias

Recebido: 04/10/04 Aceito: 19/10/05

\section{RESUMO}

Esse trabalho apresenta a avaliação de dados de operação de sistema de tratamento de águas residuárias de abatedouro de frango, através da análise de séries temporais. $\mathrm{O}$ objetivo principal foi a obtenção de modelo de previsão capaz de antecipar o controle da operação do sistema de tratamento de águas residuárias do abatedouro, constituindo subsídio para obtenção de estratégias de gestão do sistema de tratamento existente. Registros de 42 meses de parâmetros de monitoramento foram utilizados na modelagem de séries temporais. $\mathrm{O}$ modelo de ajuste exponencial para previsão dos valores de demanda química de oxigênio do efluente industrial e do afluente dos reatores biológicos apresentou bom ajuste (erro percentual absoluto da média aritmética menor que $20 \%$ ) com diferenças na capacidade de previsão menores que $15 \%$.

PALAVRAS-CHAVE: Abatedouro de frango, ajuste exponencial, análise de séries temporais, médias móveis, tratamento de águas residuárias.

\begin{abstract}
This paper presents the evaluation of the operating data of the poultry slaughterhouse wastewater treatment plant, by means of time series analysis. It aimed at obtaining a forecasting model able to have the operation of the slaughterhouse wastewater treatment system under control, what leads to subsidy for acquiring the management strategies of the wastewater treatment system. Records of 42 months of the monitoring parameters were used in the time series modeling. The exponentially weighted moving average model for obtaining chemical oxygen demand values of the industrial effluent and the reactors influent stream showed proper adjustment (mean absolute percentage error values smaller than 20\%), where the differences on the prediction feasibility were smaller than $15 \%$.
\end{abstract}

KEYWORDS: Poultry slaughterhouse, exponentially weighted moving average, time series analysis, moving average, wastewater treatment.

\section{INTRODUÇÃO}

A disposição de águas residuárias industriais em redes de esgoto ou em corpos hídricos receptores deve atender aos padrôes de lançamento estipulados por normas e regulamentaçóes, os quais estão cada vez mais restritivos. $\mathrm{O}$ não atendimento à legislação ambiental pode acarretar em sanções legais como autuaçôes e interrupções do lançamento das águas residuárias.

Para o atendimento aos restritivos padrões de lançamento são recomendados procedimentos e tecnologias de controle efetivos para os efluentes industriais. Contudo, as diferentes composições físicas, químicas e biológicas; a potencialidade de toxicidade; as variações de qualidade e de volumes gerados nos pro- cessos produtivos e os diversos pontos de geração de águas residuárias são indicativos preponderantes da necessidade de caracterizar, quantificar e tratar adequadamente os efluentes líquidos anteriormente à disposição final no meio ambiente.

As características físicas, químicas e biológicas das águas residuárias industriais são, predominantemente, depen- 
dentes do tipo da indústria, do tamanho das unidades industriais, do uso da água e da reciclagem dos efluentes líquidos.

A caracterização qualitativa e quantitativa da água residuária é realizada para determinação da concentração e da carga de qualquer constituinte, variáveis interferentes no comportamento das unidades dos sistemas de tratamento de águas residuárias (STAR). A concentração é um dos indicadores do desempenho dos processos em relação à eficiência do tratamento.

O monitoramento é fundamental para a avaliação do desempenho das unidades e as estratégias adequadas de controle protagonizam o desempenho eficiente dos STAR, estando, indubitavelmente, associadas à qualidade do efluente final. $O$ controle das operações e dos processos de tratamento visa manter os valores dos parâmetros de monitoramento dos STAR dentro dos padróes estipulados e reduzir sua variabilidade. Dessa forma, a qualidade do efluente é garantida, fazendo com que o sistema de tratamento se comporte de maneira específica.

A avaliação antecipada do comportamento do efluente industrial possibilita adotar medidas de controle que garantam desempenho adequado dos STAR. Esta pode ser uma estratégia utilizada para obtenção de parâmetros de monitoramento quando a determinação for demorada, evitando distúrbios que uma vez ocorridos não possam ser corrigidos rapidamente. O operador deve ser capaz de prever se um conjunto de condiçōes de operação pode resultar em desestabilidade do processo (Berthouex \& Box, 1996).
Uma série temporal é um conjunto de dados numéricos obtidos durante períodos regulares ao longo do tempo. A análise de séries temporais visa a formulação de modelos capazes de descrever a dependência do dado com relação ao tempo e predizer valores futuros. Uma série temporal pode ser formada pelos componentes tendência, sazonal, cíclico e aleatório (Nascimento et al, 1996; Levine et al, 2000).

A análise de séries temporais utilizada na operação de STAR (Tabela 1) pode ser ferramenta útil no controle do sistema, possibilitando ao operador atuar preventivamente em possíveis desequilíbrios no processo, pois fornece uma estimativa numérica da qualidade da água residuária com um ou mais dias de antecedência, caso não seja possível à realização do controle laboratorial. No entanto, o modelo não é capaz de predizer distúrbios causados por falhas nos equipamentos ou por introdução de compostos tóxicos. Os modelos podem ser facilmente atualizados à medida que os dados forem obtidos (Berthouex \& Box, 1996).

\section{METODOLOGIA}

\section{Sistema de tratamento de águas residuárias (STAR) da indústria de abate de frango}

A Céu Azul Alimentos Ltda localiza-se na cidade de Sorocaba, SP e está inserida na Sub-bacia Sorocaba/Pirajibu da Unidade de Gerenciamento de Recursos Hídricos - 10 do Sorocaba/Médio Tiête. O STAR é composto por pe- neiras rotativas e estática, tanque de equalização, sistema de flotação por ar dissolvido e dois reatores anaeróbios de fluxo ascendente com manta de lodo (UASB). As peneiras rotativas removem sólidos maiores que $1000 \mu \mathrm{m}$ (penas e vísceras), enquanto que a peneira estática remove sólidos finos maiores que $750 \mu \mathrm{m}$. O efluente da peneira estática e a fração líquida do sangue, após coagulação, são conduzidos ao tanque de equalização com volume de $142 \mathrm{~m}^{3}$ antes de serem encaminhados ao sistema de flotação por ar dissolvido e aos reatores UASB com volume de $450 \mathrm{~m}^{3}$ cada (Figura 1). Os reatores foram inoculados com lodo proveniente de digestor de lodo de estação de tratamento de esgotos domésticos de Barueri, SP (Del Nery et al, 2001a).

As amostras do efluente industrial (tanque de equalização) e do afluente e efluente dos reatores foram coletadas, mensalmente, ao longo de 42 meses após a partida do STAR. Alíquotas foram coletadas em intervalos de 30 minutos, durante 24 horas, preservadas em gelo, e em seguida compostas em relação à vazão de acordo com CETESB (1987 e 1991).

Os parâmetros de monitoramento demanda química de oxigênio (DQO), demanda bioquímica de oxigênio (DBO), óleos e graxas, alcalinidade total, ácidos voláteis, $\mathrm{pH}$, nitrogênio total, nitrogênio amoniacal, fosfato total e série sólidos foram analisados no Laboratório de Saneamento do Departamento de Hidráulica e Saneamento da Escola de Engenharia de São Carlos - Universidade de São Paulo (Del Nery et al, 2004) de acordo com Standard Methods for the Examination

Tabela I - Aplicações da análise de séries temporais no tratamento de águas residuárias

\begin{tabular}{|c|c|c|c|c|}
\hline Referência & Modelo & Parâmetro & $\begin{array}{l}\text { Tipo de água } \\
\text { residuária }\end{array}$ & $\begin{array}{l}\text { Sistema de tratamento } \\
\text { da água residuária }\end{array}$ \\
\hline Adeyemi et al (1979) & ARMAV $^{a}$ & Fósforo & Esgoto sanitário & $\begin{array}{l}\text { Lodos ativados com } \\
\text { remoção de fósforo }\end{array}$ \\
\hline Berthouex et al $(1976,1978)$ & Autoregressivo & DBO, SSV, TDH & Esgoto sanitário & Lodos ativados \\
\hline Berthouex \& Box (1996) & $\begin{array}{c}\text { Ajuste } \\
\text { exponencial }\end{array}$ & $\mathrm{DBO}, \mathrm{SS}$ & Esgoto sanitário & Lodos ativados \\
\hline Debelak \& Sims (1981) & ARIMA $^{b}$ & DQO & Esgoto sanitário & Lodos ativados \\
\hline Ellis et al (1993) & ARMA $^{c}$ & $\begin{array}{l}\text { DBO, nitrogênio } \\
\text { amoniacal e orgânico }\end{array}$ & Esgoto sanitário & Lodos ativados \\
\hline van Dongen \& Geuens (1998) & $\mathrm{ARMA}^{\mathrm{c}}$ & DQO, SS, SSV & Cervejaria & Lodos ativados \\
\hline
\end{tabular}





Figura I - Fluxograma do sistema de tratamento de águas residuárias do abatedouro de frango. Pontos de
amostragem: (I) Efluente industrial. (2) Afluente dos reatores. (3) Efluentes dos reatores

of Water and Wastewater (1998). O parâmetro DQO foi utilizado, nesse estudo, por ser representativo e de análise simples e rápida.

\section{Análise de séries temporais}

Foram ajustados os modelos de médias móveis e de ajuste exponencial aos dados operacionais de DQO.

Modelo das médias móveis

Para um conjunto de dados:

$\mathrm{Y}_{1}, \mathrm{Y}_{2}, \mathrm{Y}_{3}, \ldots$

define-se uma média móvel de ordem $\mathrm{N}$, que é obtida pela seqüência das médias aritméticas:

$\frac{\mathrm{Y}_{1}+\mathrm{Y}_{2}+\ldots+\mathrm{Y}_{\mathrm{N}}}{\mathrm{N}}, \frac{\mathrm{Y}_{2}+\mathrm{Y}_{3}+\ldots+\mathrm{Y}_{\mathrm{N}+1}}{\mathrm{~N}}$,
$, \frac{\mathrm{Y}_{3}+\mathrm{Y}_{4}+\ldots+\mathrm{Y}_{\mathrm{N}+2}}{\mathrm{~N}}, \ldots$

As somas dos numeradores de (2) são denominados totais móveis de ordem $\mathrm{N}$.

Mediante o emprego das médias móveis de ordem apropriada, podem ser eliminadas as variações sazonais, cíclicas e irregulares (Morettin \& Toloi, 1986; Spiegel, 1993).

\section{Modelo do ajuste exponencial}

O método do ajuste exponencial (equação 3) fornece média móvel exponencialmente ponderada ao longo de toda a série. Cada previsão ou cálculo de ajuste depende de todos os valores previamente observados. Com o ajuste exponencial, os pesos designados para os valores observados decrescem com o tempo, de modo que, quando se efetua o cálculo, o valor observado mais recentemente recebe o maior peso, o valor observado anterior- mente recebe o segundo maior peso, e assim por diante.

$$
\mathrm{E}_{\mathrm{i}}=\mathrm{W} \mathrm{Y}_{\mathrm{i}}+(1-\mathrm{W}) \mathrm{E}_{\mathrm{i}-1}
$$

sendo: $\mathrm{E}_{\mathrm{i}}$ o valor da série exponencialmente ajustada calculada no período de tempo $\mathrm{i}, \mathrm{E}_{\mathrm{i}-1}$ o valor da série exponencialmente ajustada já calculada no período de tempo i-1, $\mathrm{Y}_{\mathrm{i}}$ o valor observado da série temporal no período i e W o coeficiente de ajuste $(0<\mathrm{W}<1)$.

Uma série de médias móveis de ordem $\mathrm{N}$ está relacionada à série ajustada exponencialmente conforme equação (4):

$\mathrm{W}=\frac{2}{\mathrm{~N}+1}$

Para ajuste de série, eliminando variaçôes cíclicas e irregulares indesejáveis, deve-se selecionar um valor baixo para o coeficiente de ajuste, próximo a zero, assim as tendências gerais de longo prazo da série ficarão aparentes. Para previsão, deve-se escolher um valor alto para o coeficiente de ajuste, próximo a um, em que os direcionamentos futuros de curto prazo podem ser previstos de maneira mais adequada.

Para utilizar a média móvel exponencialmente ponderada para fins de previsão, o valor ajustado no período de tempo atual, período i, é utilizado como estimativa projetada do valor observado da série temporal, no período de tempo seguinte, $i+1$ (Levine et al, 2000):

$$
\begin{aligned}
& \hat{\mathrm{Y}}_{\mathrm{i}+1}=\mathrm{E}_{\mathrm{i}} \\
& \hat{\mathrm{Y}}_{\mathrm{i}+1}=\mathrm{W} \mathrm{Y}_{\mathrm{i}}+(1-\mathrm{W}) \hat{\mathrm{Y}}_{\mathrm{i}}
\end{aligned}
$$

\section{Escolha do modelo de previsão apropriado}

A escolha do modelo de previsão apropriado foi realizada baseada na análise de resíduos (Figura 2) e na medida do erro residual.

$$
e_{i}=\left(Y_{i}-\hat{Y_{i}}\right)
$$

As diferenças entre os dados observados e os dados ajustados são conhecidas como resíduos. Para o $i$-ésimo valor na série temporal de n observaçôes, o resíduo é definido como:

sendo $\mathrm{Y}_{\mathrm{i}} \mathrm{o}$ valor observado no período i e $\hat{Y}_{\mathrm{i}}$ o valor ajustado no período i.

Caso o modelo em questão se ajuste adequadamente, os resíduos representam o componente irregular da série temporal, e devem, portanto ser distribuídos aleatoriamente ao longo da série (Figura 2a). Caso o modelo não se ajuste adequadamente, os resíduos podem demonstrar um padrão sistemático, deixando, por exemplo, de considerar uma tendência (Figura 2b), uma variação cíclica (Figura 2c) ou variações sazonais (Figura 2d) (Levine et al, 2000).

A magnitude do erro residual foi avaliada pelo erro percentual absoluto da média aritmética (EPAM) e pelo desvio absoluto da média aritmética (DAM).

O EPAM avalia a exatidão dos valores ajustados da série temporal, expresso em porcentagem.

EPAM $=\frac{\sum\left|\left(Y_{i}-\hat{Y}_{i}\right) / Y_{i}\right|}{n} \times 100 \quad\left(Y_{i} \neq 0\right)$

sendo: $\mathrm{Y}_{\mathrm{i}}$ o valor real, $\hat{\mathrm{Y}}_{\mathrm{i}}$ o valor ajustado e n o número de observaçóes. 


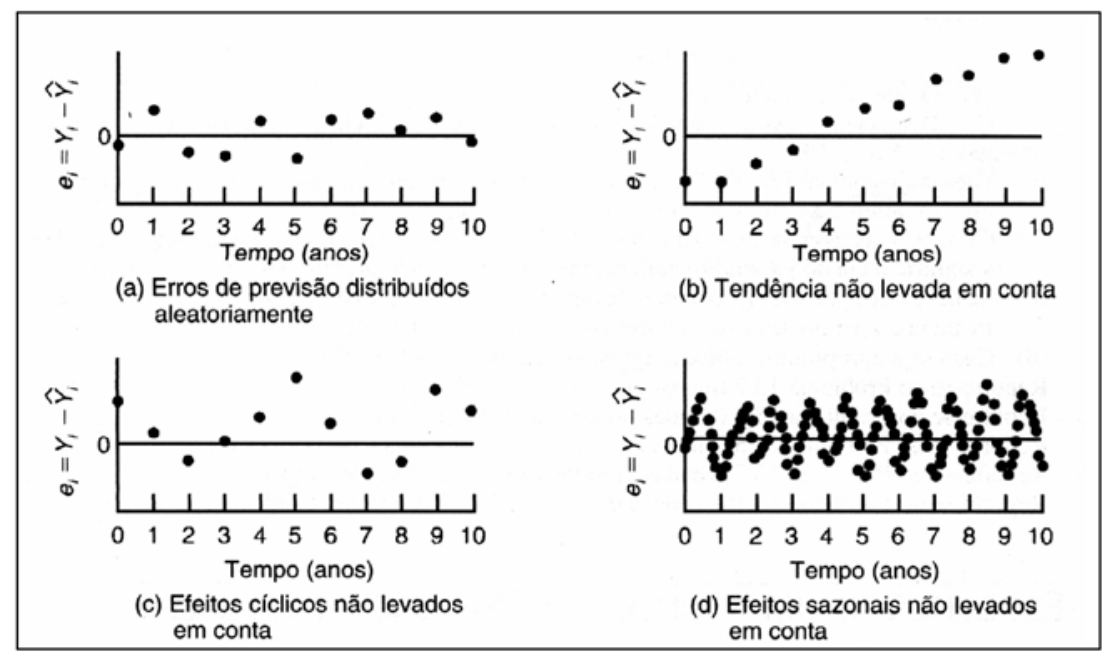

Figura 2 - Análise de resíduos para avaliar padrões de erros (Levine et al, 2000)

O ajuste do modelo pode ser classificado como excelente, bom, aceitável ou ruim para valores de EPAM $<10 \%, 10 \%$ a $20 \%, 20 \%$ a $50 \%$ e $>50 \%$, respectivamente (Lin, 1998).

O DAM também avalia a exatidão dos valores ajustados da série temporal, porém o seu valor é expresso na mesma unidade dos dados. O modelo de previsão mais apropriado é o que apresenta o menor valor de DAM (Levine et al, 2000).

$D A M=\frac{\sum_{i=1}^{n}\left|Y_{i}-\hat{Y}_{i}\right|}{n}$

sendo: $\mathrm{Y}_{\mathrm{i}}$ o valor real, $\mathrm{Y}_{\mathrm{i}}$ o valor ajustado e n o número de observaçóes.

\section{RESULTADOS E DISCUSSÃO}

\section{Estatística descritiva}

A Tabela 2 apresenta os valores médios de DQO do efluente industrial e do afluente dos reatores UASB com seus respectivos desvios-padróes, valores mínimos e máximos obtidos, mensalmente, ao longo de 42 meses de operação, após a partida do STAR. Os histogramas de DQO (Figura 3) mostram condição de simetria das distribuições de freqüência, indicando distribuição normal.

A Figura 4 mostra as representações gráficas das séries temporais dos dados de DQO. A análise das curvas sugere que não há efeito de tendência. Dessa forma, podese empregar o método das médias móveis ou o método de ajuste exponencial para ajustar a série.
Tabela 2 - Sumário dos dados de DQO do STAR

\begin{tabular}{ccccc}
\hline Ponto de Amostragem & N & Média & Mínimo & Máximo \\
\hline Efluente industrial & 42 & $3730 \pm 809$ & 2125 & 6085 \\
Afluente dos reatores & 42 & $2312 \pm 588$ & 1134 & 3870 \\
\hline
\end{tabular}

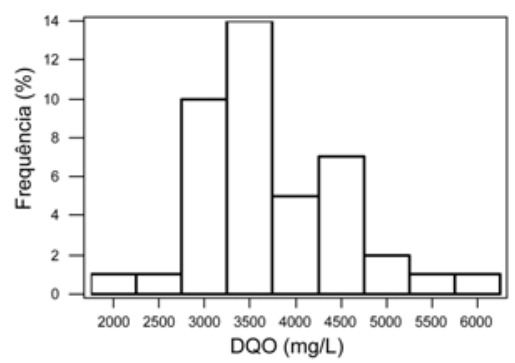

(a)

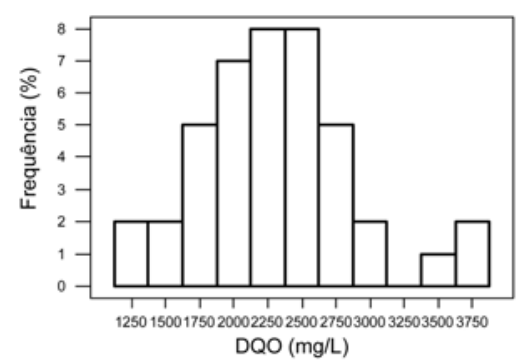

(b)
Figura 3 - Histograma para DQO. (a) Efluente industrial. (b) Afluente dos reatores

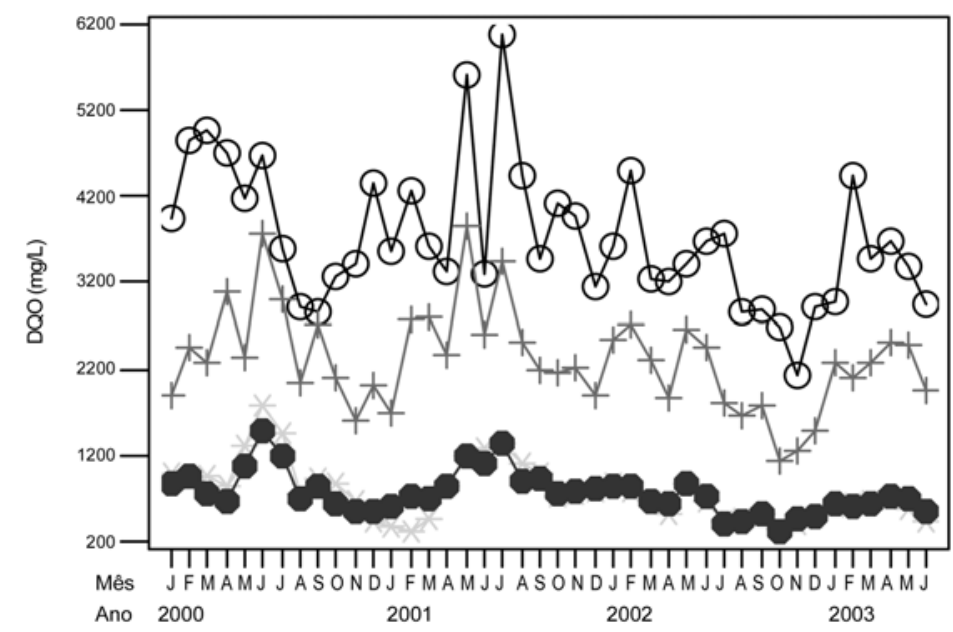

Figura 4 - Gráfico de séries temporais para DQO. o Efluente industrial, + Afluente dos reatores, * Efluente do Reator A, E Efluente do Reator $B$ 
Ajuste de modelos para o efluente industrial utilizando o parâmetro DQO

Os modelos de médias móveis de $3^{\mathrm{a}}, 5^{\mathrm{a}}$ e $7^{\mathrm{a}}$ ordens e de ajuste exponencial, adotando-se valores para o coeficiente de ajuste de 0,27 e 0,5 , foram ajustados aos dados (Tabela 3).

A partir da análise de resíduos (Figura 5b), dos valores de EPAM e de DAM foi escolhido o modelo de ajuste exponencial para $\mathrm{W}=0,27$, pois apresentou os resíduos aleatoriamente distribuídos ao longo da série e os menores valores de EPAM, 16\%, e DAM, $579 \mathrm{mg} / \mathrm{L}$. A Figura 5a apresenta a curva dos dados originais e do ajuste exponencial.

\section{Ajuste de modelos para o afluente dos reatores utilizando o parâmetro DQO}

Os modelos de médias móveis de $3^{\mathrm{a}}, 5^{\mathrm{a}} \mathrm{e} 7^{\mathrm{a}}$ ordens e de ajuste exponencial, adotando-se valores para o coeficiente de ajuste de 0,27 e 0,46 , foram ajustados aos dados (Tabela 4).

A partir da análise de resíduos e dos valores de EPAM e de DAM foi escolhido o modelo de ajuste exponencial para $\mathrm{W}=0,46$, pois apresentou os resíduos aleatoriamente distribuídos ao longo da série (Figura 6b) e os menores valores de EPAM, 20\%, e DAM, $459 \mathrm{mg} / \mathrm{L}$. A Figura 6a apresenta a curva dos dados originais e do ajuste exponencial.

\section{Estimativa de previsões}

A partir da scolha do modelo que melhor se ajustou aos dados, foram feitas análises de previsão, com intervalo de confiança de $95 \%$, para o valor seguinte e comparado com o valor obtido da análise em laboratório.

Pelo modelo de ajuste exponencial para $\mathrm{W}=0,27$, o valor de previsão da DQO do efluente industrial para o mês seguinte foi de $3304 \mathrm{mg} / \mathrm{L}$, com limite inferior de $1885 \mathrm{mg} / \mathrm{L}$ e limite superior de $4722 \mathrm{mg} / \mathrm{L}$. O valor medido no mês, que não entrou na análise de séries temporais, foi de $2830 \mathrm{mgDQO} / \mathrm{L}$, representando uma diferença de $14 \%$ do valor previsto pelo modelo em relação ao valor medido.

Pelo modelo de ajuste exponencial para $\mathrm{W}=0,46$, o valor de previsão $\mathrm{da}$
Tabela 3 - Médias móveis e ajuste exponencial para DQO do efluente industrial do abatedouro

\begin{tabular}{|c|c|c|c|c|c|c|}
\hline \multirow[t]{2}{*}{ Mês } & \multirow[t]{2}{*}{$\mathrm{DQO}(\mathrm{mg} / \mathrm{L})$} & \multicolumn{3}{|c|}{ Médias móveis } & \multicolumn{2}{|c|}{ Ajuste operacional } \\
\hline & & $\mathrm{N}=3$ & $\mathrm{~N}=5$ & $\mathrm{~N}=7$ & $\mathrm{~W}=0,27$ & $\mathrm{~W}=0,5$ \\
\hline 1 & 3960 & * & * & * & 4233 & 4260 \\
\hline 2 & 4860 & 4593 & * & $*$ & 4401 & 4560 \\
\hline 3 & 4960 & 4847 & 4536 & * & 4551 & 4760 \\
\hline 4 & 4720 & 4620 & 4680 & 4421 & 4596 & 4740 \\
\hline 5 & 4180 & 4527 & 4425 & 4274 & 4485 & 4460 \\
\hline 6 & 4680 & 4148 & 4019 & 3987 & 4537 & 4570 \\
\hline 7 & 3585 & 3732 & 3646 & 3747 & 4282 & 4078 \\
\hline 8 & 2930 & 3124 & 3465 & 3562 & 3920 & 3504 \\
\hline 9 & 2856 & 3020 & 3214 & 3589 & 3635 & 3180 \\
\hline 10 & 3275 & 3185 & 3371 & 3429 & 3538 & 3227 \\
\hline 11 & 3425 & 3690 & 3497 & 3527 & 3508 & 3326 \\
\hline 12 & 4370 & 3785 & 3780 & 3625 & 3739 & 3848 \\
\hline 13 & 3560 & 4067 & 3849 & 3694 & 3691 & 3704 \\
\hline 14 & 4270 & 3817 & 3832 & 4029 & 3846 & 3987 \\
\hline 15 & 3620 & 3743 & 4082 & 4010 & 3786 & 3804 \\
\hline 16 & 3340 & 4193 & 4028 & 4255 & 3666 & 3572 \\
\hline 17 & 5620 & 4083 & 4391 & 4381 & 4190 & 4596 \\
\hline 18 & 3290 & 4998 & 4555 & 4268 & 3949 & 3943 \\
\hline 19 & 6085 & 4605 & 4583 & 4341 & 4521 & 5014 \\
\hline 20 & 4440 & 4668 & 4285 & 4432 & 4499 & 4727 \\
\hline 21 & 3480 & 4017 & 4423 & 4080 & 4226 & 4103 \\
\hline 22 & 4130 & 3863 & 3837 & 4128 & 4200 & 4117 \\
\hline 23 & 3980 & 3755 & 3674 & 3903 & 4141 & 4048 \\
\hline 24 & 3155 & 3587 & 3880 & 3731 & 3877 & 3602 \\
\hline 25 & 3625 & 3763 & 3702 & 3691 & 3810 & 3613 \\
\hline 26 & 4510 & 3792 & 3546 & 3591 & 3997 & 4062 \\
\hline 27 & 3240 & 3650 & 3601 & 3547 & 3794 & 3651 \\
\hline 28 & 3200 & 3290 & 3610 & 3636 & 3635 & 3425 \\
\hline 29 & 3430 & 3433 & 3463 & 3528 & 3580 & 3428 \\
\hline 30 & 3670 & 3625 & 3389 & 3297 & 3604 & 3549 \\
\hline 31 & 3776 & 3439 & 3328 & 3217 & 3650 & 3662 \\
\hline 32 & 2870 & 3179 & 3178 & 3063 & 3441 & 3266 \\
\hline 33 & 2892 & 2814 & 2869 & 2993 & 3294 & 3079 \\
\hline 34 & 2680 & 2566 & 2700 & 2893 & 3129 & 2880 \\
\hline 35 & 2125 & 2580 & 2721 & 2990 & 2860 & 2502 \\
\hline 36 & 2935 & 2678 & 3033 & 3077 & 2880 & 2719 \\
\hline 37 & 2975 & 3453 & 3193 & 3189 & 2906 & 2847 \\
\hline 38 & 4450 & 3635 & 3504 & 3289 & 3320 & 3648 \\
\hline 39 & 3480 & 3870 & 3593 & 3409 & 3363 & 3564 \\
\hline 40 & 3680 & 3513 & 3590 & * & 3448 & 3622 \\
\hline 41 & 3380 & 3340 & * & * & 3429 & 3501 \\
\hline 42 & 2960 & * & * & * & 3304 & 3231 \\
\hline EPAM (\%) & & 17 & 18 & 18 & 16 & 16 \\
\hline $\mathrm{DAM}(\mathrm{mg} / \mathrm{L})$ & & 620 & 648 & 647 & 579 & 594 \\
\hline
\end{tabular}


Nardi, I. R. et al.

Tabela 4 - Médias móveis e ajuste exponencial para DQO afluente dos reatores

\begin{tabular}{|c|c|c|c|c|c|c|}
\hline \multirow[t]{2}{*}{ Mês } & \multirow[t]{2}{*}{ DQO $(\mathrm{mg} / \mathrm{L})$} & \multicolumn{3}{|c|}{ Médias móveis } & \multicolumn{2}{|c|}{ Ajuste operacional } \\
\hline & & $\mathrm{N}=3$ & $\mathrm{~N}=5$ & $\mathrm{~N}=7$ & $\mathrm{~W}=0,27$ & $\mathrm{~W}=0,46$ \\
\hline 1 & 1890 & * & $*$ & $*$ & 2435 & 2083 \\
\hline 2 & 2460 & 2207 & * & $*$ & 2442 & 2255 \\
\hline 3 & 2270 & 2607 & 2408 & * & 2396 & 2262 \\
\hline 4 & 3090 & 2564 & 2786 & 2690 & 2583 & 2641 \\
\hline 5 & 2332 & 3067 & 2896 & 2711 & 2515 & 2500 \\
\hline 6 & 3780 & 3041 & 2850 & 2748 & 2857 & 3085 \\
\hline 7 & 3010 & 2942 & 2776 & 2725 & 2898 & 3051 \\
\hline 8 & 2036 & 2589 & 2730 & 2514 & 2665 & 2587 \\
\hline 9 & 2720 & 2287 & 2297 & 2470 & 2680 & 2648 \\
\hline 10 & 2104 & 2146 & 2101 & 2170 & 2525 & 2399 \\
\hline 11 & 1615 & 1916 & 2029 & 2139 & 2279 & 2040 \\
\hline 12 & 2028 & 1774 & 2043 & 2247 & 2211 & 2035 \\
\hline 13 & 1680 & 2166 & 2182 & 2198 & 2068 & 1872 \\
\hline 14 & 2790 & 2422 & 2334 & 2450 & 2263 & 2292 \\
\hline 15 & 2795 & 2653 & 2702 & 2590 & 2406 & 2522 \\
\hline 16 & 2375 & 3013 & 2885 & 2792 & 2398 & 2455 \\
\hline 17 & 3870 & 2947 & 3015 & 2912 & 2795 & 3102 \\
\hline 18 & 2595 & 3302 & 2960 & 2827 & 2741 & 2870 \\
\hline 19 & 3442 & 2852 & 2923 & 2737 & 2931 & 3132 \\
\hline 20 & 2520 & 2717 & 2582 & 2713 & 2820 & 2852 \\
\hline 21 & 2190 & 2292 & 2505 & 2432 & 2650 & 2549 \\
\hline 22 & 2165 & 2188 & 2198 & 2425 & 2519 & 2373 \\
\hline 23 & 2210 & 2093 & 2203 & 2321 & 2435 & 2299 \\
\hline 24 & 1904 & 2220 & 2307 & 2291 & 2292 & 2118 \\
\hline 25 & 2546 & 2387 & 2337 & 2243 & 2361 & 2314 \\
\hline 26 & 2710 & 2524 & 2266 & 2313 & 2455 & 2495 \\
\hline 27 & 2315 & 2293 & 2415 & 2347 & 2417 & 2413 \\
\hline 28 & 1854 & 2273 & 2395 & 2332 & 2265 & 2157 \\
\hline 29 & 2650 & 2317 & 2214 & 2207 & 2369 & 2383 \\
\hline 30 & 2448 & 2300 & 2085 & 2075 & 2390 & 2413 \\
\hline 31 & 1801 & 1974 & 2071 & 1906 & 2231 & 2133 \\
\hline 32 & 1672 & 1752 & 1767 & 1818 & 2080 & 1922 \\
\hline 33 & 1782 & 1529 & 1526 & 1654 & 2000 & 1858 \\
\hline 34 & 1134 & 1385 & 1466 & 1629 & 1766 & 1527 \\
\hline 35 & 1240 & 1292 & 1587 & 1672 & 1624 & 1396 \\
\hline 36 & 1502 & 1672 & 1650 & 1759 & 1591 & 1444 \\
\hline 37 & 2275 & 1959 & 1879 & 1862 & 1776 & 1824 \\
\hline 38 & 2100 & 2217 & 2132 & 2054 & 1863 & 1950 \\
\hline 39 & 2277 & 2294 & 2327 & 2154 & 1975 & 2100 \\
\hline 40 & 2505 & 2421 & 2261 & * & 2118 & 2285 \\
\hline 41 & 2480 & 2309 & * & * & 2216 & 2374 \\
\hline 42 & 1942 & * & * & * & 2142 & 2177 \\
\hline EPAM (\%) & & 21 & 25 & 26 & 22 & 20 \\
\hline DAM (mg/L) & & 477 & 540 & 539 & 472 & 459 \\
\hline
\end{tabular}




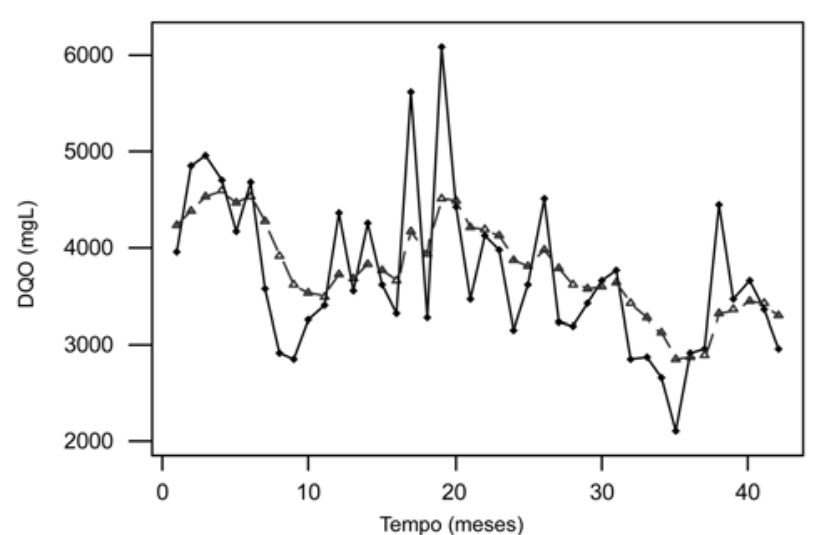

(a)



(b)

Figura 5: (a) Gráfico da série exponencialmente ajustada $(W=0,27)$ para $D Q O$ do efluente industrial. $\checkmark$ Dados originais, $\triangle$ Ajuste exponencial. (b) Análise de resíduos.

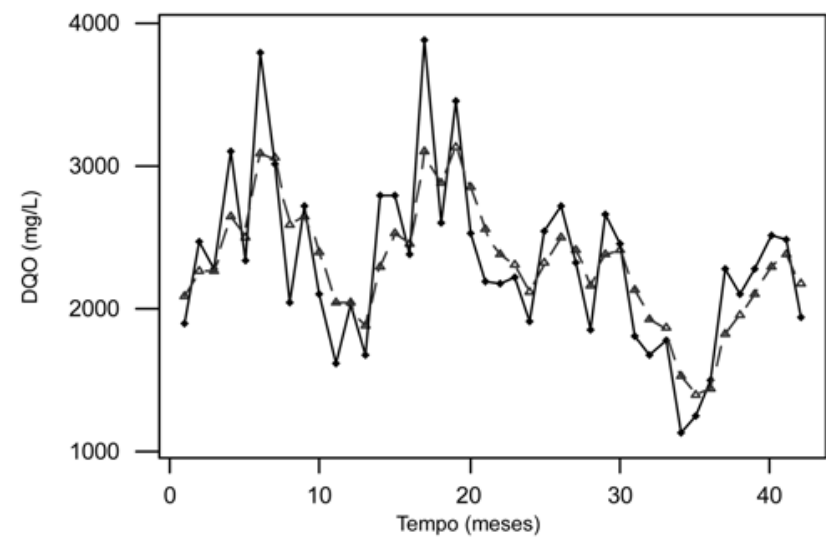

(a)

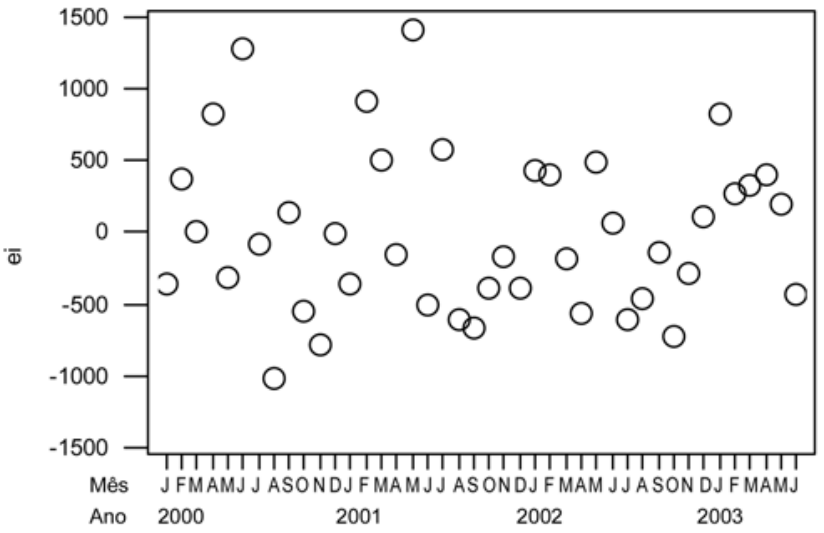

(b)

Figura 6 - (a) Gráfico da série exponencialmente ajustada $(W=0,46)$ para $D Q O$ do afluente dos reatores. $\checkmark$ Dados originais, $\triangle$ Ajuste exponencial. (b) Análise de resíduos

DQO do afluente dos reatores para o mês seguinte foi de $2177 \mathrm{mg} / \mathrm{L}$, com limite inferior de $1052 \mathrm{mg} / \mathrm{L}$ e limite superior de $3301 \mathrm{mg} / \mathrm{L}$. O valor medido no mês, que não entrou na análise de séries temporais, foi de $1845 \mathrm{mgDQO} / \mathrm{L}$, representando uma diferença de $15 \%$ do valor previsto pelo modelo em relação ao valor medido.

Os valores previstos estão de acordo com a variabilidade do efluente industrial e do afluente dos reatores. A variação da qualidade do efluente industrial está principalmente associada ao processo industrial: quantidade de aves abatidas, vazão de água utilizada, procedimentos operacionais e de higienização de equipamentos e instalaçôes. Além desses fatores, a qualidade do afluente dos reatores está relacionada à eficiência do sistema de flotação por ar dissolvido.
É importante que essas projeções sejam examinadas tão logo um novo valor do parâmetro seja observado, sendo comparado com a sua projeção. Se a diferença for muito grande, o modelo de previsão deve ser revisado (Levine et al, 2000).

\section{CONCLUSÕES}

O modelo de ajuste exponencial para previsão dos valores de DQO do efluente industrial e do afluente dos reatores foi o que apresentou melhor ajuste aos dados de monitoramento do STAR do abatedouro de frango (EPAM < 20\%). A capacidade de previsão do modelo apresentou diferença em torno de $15 \%$ do valor previsto em relação ao valor real.

\section{AGRADECIMENTOS}

Os autores agradecem a FAPESP Fundação de Amparo à Pesquisa do Estado de São Paulo pelo apoio financeiro e bolsas de pesquisa concedidos a este projeto (processo n ${ }^{\circ}$ 2002/03997-1) e a Céu Azul Alimentos Ltda pelo apoio operacional.

\section{REFERÊNCIAS}

ADEYEMI, S. O.; WU, S. M.; BERTHOUEX, P. M. Modeling and control of a phosphorus removal process by multivariate time series method. Water Research, v.13, p.105-112. 1979.

AMERICAN PUBLIC HEALTH ASSOCIATION (APHA); AMERICAN WATER WORKS ASSOCIATION (AWWA); WATER ENVIRONMENT FEDERATION (WEF). Standard methods for the examination 
of water and wastewater. 20 ed. Washington DC. APHA-WEF, 1195p. 1998.

AMORIM, A.K.B.; DE NARDI, I.R.; DEL NERY, V. Minimization of industrial effluents: case study of a poultry slaughterhouse. In SIMPOSIO INTERNAZIONALE DI INGEGNERIA SANITARIA AMBIENTALE, 7, Taormina. CD ROM. Taormina: Edizion CSISA, 2004.

BERTHOUEX, P. M. et al. The use of stochastic models in the interpretation of historical data from sewage treatment plants. Water Research, v.10, p.689-698. 1976.

BERTHOUEX, P. M.; HUNTER, W. G.; PALLESEN L. Dynamic behavior of an activated sludge plant. Water Research, v.12, p.957-972. 1978

BERTHOUEX, P. M.; BOX, G. E. Time series models for forecasting wastewater treatment plant performance. Water Research, v.30, n.8, p.1865-1875. 1996.

CETESB Guia de coleta e preservação de amostras de água. São Paulo. CETESB, 150p. 1987.

CETESB Avaliação de desempenho de estaçôes de tratamento de esgotos. São Paulo. CETESB, 38p. 1991.

DEBELAK, K. A.; SIMS, C. A. Stochastic modeling of an industrial activated sludge process. Water Research, v.15, p.1173-1183. 1981.

DEL NERY, V.; DAMIANOVIC, M. H. R. Z.; BARROS, F. G. The use of upflow anaerobic sludge blanket reactors in the treatment of poultry slaughterhouse wastewater. Water Science and Technology, v.44, p.83-88. 2001a.

DEL NERY, V. et al. Upflow anaerobic sludge blanket reactors treating poultry slaughterhouse wastewater under different design and operational parameters. In: WORLD CONGRESS ANAEROBIC DIGESTION, 9, Antuérpia. Anais. Antuérpia: Technological Institute, p.423-427. $2001 \mathrm{~b}$.

DEL NERY, V. et al. Poultry slaughterhouse wastewater treatment plant performance over a long-term operation. In: THE FIRST INTERNATIONAL MEETING ON ENVIRONMENTAL BIOTECHNOLOGY AND ENGINEERING, 1, Cidade do México. CD ROM. Cidade do México: CINVESTAV, 2004

ELLIS, G. W.; GRASSO, D.; GE, X. $A R M A$ processes and reliability-based design of wastewater-treatment facilities. Journal of Environmental Engineering, v.119, n.3, p.463-477. 1993

LEVINE, D. M.; BERENSON, M. L.; STEPHAN, D. Estatística: teoria e aplicações

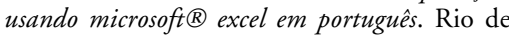
Janeiro. Livros Técnicos e Científicos Editora, 811 p. 2000

LIN, J. G. Feasibility study on prediction of properties of municipal solid waste with time series models. Journal of Hazardous Materials, v., p.47-57. 1998.
MORETTIN, P. A.; TOLOI, C. M. C. Séries temporais. 2ed. São Paulo. Atual Editora. 136p. 1986

NASCIMENTO, N. O. et al. Investigação cientifica em engenharia sanitária e ambiental. Parte 3: Análise estatís-tica de dados e de modelos. Engenharia Sanitária e Ambiental, v.4, p.152-168, Out./Dez. 1996.

SPIEGEL, M. R. Estatística: resumo da teoria, 383 problemas resolvidos, 416 problemas suplementares. 3 ed. São Paulo. McGraw-Hill do Brasil. 643p. 1993.

VAN DONGEN, G.; GEUENS, L. Multivariate time series analysis for design and operation of a biological wastewater treatmen plant. Water Research, v.32, n.3, p.691-700. 1998

\section{Endereço para correspondência:}

\section{Ivana Ribeiro de Nardi Centro Universitário Central \\ Paulista}

Rua Miguel Petroni, 5 I I I

I3563-470 São Carlos - SP - Brasil

Tel.: (I6) 3307-2 I I I

E-mail: ivananardi@unicep.com.br

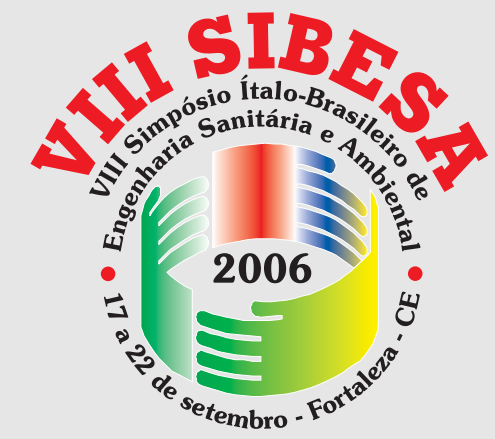

Recebimento de resumos 06/01/06

\section{Comunicação aos autores} 15/04/06

Envio de trabalhos completos 30/05/06 